\title{
An Enhanced Partial Transmit Sequence Segmentation Schemes to Reduce the PAPR in OFDM Systems
}

\author{
Yasir Amer Al-Jawhar and Nor \\ Shahida M. Shah \\ Dept. of Communication \\ Faculty of Electrical and Electronic \\ Engineering \\ Universiti Tun Hussein Onn \\ Malaysia \\ Batu Pahat, Johor, Malaysia
}

\author{
Montadar Abas Taher \\ Dept. of Communications \\ Engineering \\ College of Engineering \\ University of Diyala \\ Ba'aqubah, Diyala, Iraq
}

\author{
Mustafa Sami Ahmed and \\ Khairun N. Ramli \\ Dept. of Communication \\ Faculty of Electrical and Electronic \\ Engineering \\ Universiti Tun Hussein Onn \\ Malaysia \\ Batu Pahat, Johor, Malaysia
}

\begin{abstract}
Although the orthogonal frequency division multiplexing system (OFDM) is widely used in high-speed data rate wire and wireless environment, the peak-to- average-powerratio (PAPR) is one of its major obstacles for the real applications. The high PAPR value leads some devices of the OFDM system such as power amplifiers and analog to digital converters to work out of band of these devices. Thus the system efficiency is degraded. Many techniques have been proposed to overcome the high PAPR in OFDM systems such as partial transmit sequences (PTS), selected mapping and interleaving technique. PTS is considered as one of the effective PAPR reduction methods; this scheme depends on segmentation of the input data into several subblocks and then combined again. The three well-known segmentation schemes are pseudo-random, adjacent and interleaving; each scheme has PAPR reduction performance, and computational complexity differs from one to another. In this paper, five types of segmentation schemes are proposed to improve the PAPR reduction execution including sine and cosine shape as well as hybrid interleaving and adjacent schemes in new approaches. From the simulation results, the proposed methods can achieve PAPR reduction performance greater than that of the adjacent and interleaving partition schemes, without increasing the computational complexity of the system. Moreover, the enhanced schemes can realize better PAPR performance with any number of subcarriers.
\end{abstract} PTS

Keywords-OFDM; PAPR; PTS; adjacent PTS; interleaving

\section{INTRODUCTION}

Orthogonal frequency division multiplexing (OFDM) is widely used in the high-speed data rate communication environment. This is because of the OFDM system provides many advantages such as its robustness to multipath fading, immunity to inter-symbol interference (ISI), the ability for high data transmission rate, and bandwidth efficiency [1] [2] [3] [4]. OFDM system has been considered as a multiplexing technique for many digital communication systems such as wireless local area network (WLAN) IEEE.802.11 $/ \mathrm{b} / \mathrm{g} / \mathrm{n}$ [5], worldwide interoperability for microwave access IEEE.802.16 [6], digital video broadcasting (DVB) [7], and digital audio

The authors would like to thank to University Tun Hussein Onn Malaysia (UTHM) for sponsoring this work under Research Supporting Grant Scheme (RSGS) Vot No. U 102. broadcasting (DAB) [8]. Also, OFDM system is used in LongTerm Evaluation (LTE) which is the standard for the fourth generation (4G) technology [9] [10]. Moreover, because of its distinct features, OFDM is considered as an attractive candidate for the fifth generation (5G) of the mobile communication systems [11] [12]. However, the main disadvantage of the OFDM system is the high peak-to-average power ratio (PAPR), which leads to out-of-band radiation and in-band distortion. This is because the nonlinearity of the high power amplifier (HPA) at the transmitter. Hence, the spectral inefficiency and the bit error rate (BER) of the system are increased [13].

In order to reduce the PAPR, many methods have been proposed, such as clipping [14] [15], clipping and filtering [16], coding techniques [17], selective mapping (SLM) [18] [19], partial transmit sequence (PTS) [20] and tone injection [21]. Among these methods PTS is an effective technique, and it can improve the PAPR reduction performance. The principle idea of the PTS method is based on partitioning the input data into several subblocks, and then each subblock weights by the phase rotation factors before combining the subblocks again. The OFDM signal which has the lowest PAPR value is selected for transmission. Therefore, PTS technique depends on segmentation schemes and, phase rotation factors.

In literature, several scenarios have been proposed to modify the ordinary PTS method in terms of the segmentation schemes such as (Hong 2013) [22] and (Zeyid 2014) [12], where combining two types of the partitioning schemes is proposed. Reference [10] ( Jawhar 2016) introduced a new method by combining two types of segmentation schemes. In [23] and [24], Xia analyzed the coloration between the subcarriers within the subblocks of PTS technique; he concluded that the PAPR reduction depends on the subcarriers correlation and it decided by subblocks segmentation and phase rotation factors, while it is unconcerned with the input data sequence. Furthermore, Xiao in 2007 [25] improved the PAPR interpretation by reduce the correlation among the candidate signals in the time-domain. On the other hand, the authors in the [26] combined random and interleaving 
segmentation to enhance the PAPR performance, while Singh in [27] proposed hybrid combination of PTS and SLM in order to achieve better PAPR execution.

In this paper, new partition schemes are proposed to improve the PAPR performance of the PTS technique in OFDM system. Moreover, the adjacent segmentation scheme and interleaving segmentation scheme are combined in a new approach. Furthermore, the proposed methods are compared with ordinary PTS method. The proposed PTS methods can significantly enhance the PAPR performance without increasing the computational complexity.

This paper is organized as follows: Section II illustrates the PAPR problem. Section III discusses the ordinary PTS method. The PTS segmentation schemes are analyzed in Section IV. The proposed method introduced in Section V. Simulation results and discussions are given in section VI. Finally, the conclusions have been written in section VII.

\section{OFDM SYSTEM}

In OFDM system, the input data block is mapped by one of the mapping techniques such as, phase shift keying (PSK) and quadrature amplitude modulation (QAM). The data symbols transmitted in parallel to generate the frequency domain signal, where $N$ is the number of subcarriers. The discrete time domain signal can be generated by applying $N$-point inverse fast Fourier transform (IFFT) on $X_{k}$, which can be expressed as [28]

$$
x(n)=\frac{1}{\sqrt{N}} \sum_{k=0}^{N-1} X_{k} e^{j 2 \pi k \frac{n}{N}}, 0 \leq n \leq N-1
$$

where $j=\sqrt{-1}$.

The PAPR is the ratio of the maximum peak power signal to average power of OFDM signal, which can be written as [29]

$$
\begin{array}{r}
\max |x(n)|^{2} \\
P A P R=\frac{0 \leq n \leq N-1}{P_{a v} x(n)} \\
P_{a v} x(n)=E\left\{|x(n)|^{2}\right\}
\end{array}
$$

where $E\{$.$\} represents the average power of the OFDM$ signal. When the subcarriers in the time domain have the same phases, the peaks of the subcarriers are added together constructively, and thus lead to increasing in the PAPR value of the OFDM signal.

The complementary cumulative distribution function (CCDF) is used to evaluate the distribution PAPR performance. The CCDF denotes the probability that the PAPR of the OFDM signal exceeding the threshold value, which can be defined as [30]

$$
\operatorname{Pr}\left(P A P R>P A P R_{0}\right)=1-\left(\exp \left(-P A P R_{0}\right)\right)^{N}
$$

where the $P A P R_{0}$ is the threshold value. To catch some peaks of the signal that do not appear in the PAPR calculation, oversampling is employed in the discrete baseband signal by inserting $(L-1) N$ zeros to the OFDM signal. Therefore, the $\mathrm{CCDF}$ of the continuous OFDM signal is given as [31]

$$
\operatorname{Pr}\left(P A P R>P A P R_{0}\right)=1-\left(\exp \left(-P A P R_{0}\right)\right)^{N L}
$$

where $L$ is the oversampling factor.

\section{CONVEntional Partial Transmit SEQUENCE (C- PTS)}

C-PTS technique proposed to reduce the PAPR value of the OFDM system. Fig. 1 illustrates the block diagram of the PTS method, in which the input data sequence $X_{k}$ is partitioned into $V$ non-overlapping subblocks. In each subblock, only $N / V$ samples are assigned, and the rest samples are set to zero. Therefore, the subblock $X_{v}$ is represented

$$
X=\sum_{v=1}^{V} X_{v}
$$

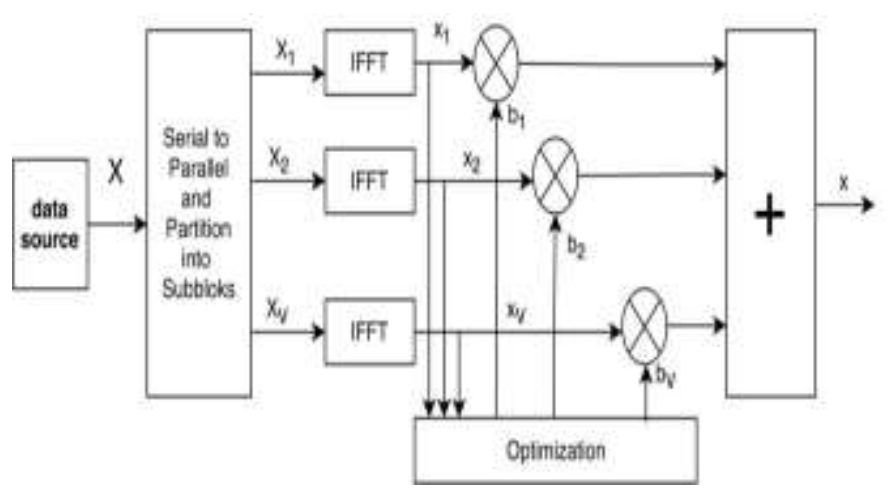

Fig. 1. PTS block diagram

The partitioned subblocks $X_{v}$ is transformed from the frequency domain into the time domain by applying IFFT operation. After that, each subblock rotates by phase weighting vectors $b_{v}$, and combines with other subblocks to generate a set of candidates. The candidate sequence which achieves the minimum PAPR value is selected for transmission [32]. Therefore, the time domain signal after combining the subblocks is given by

$$
x=\operatorname{IFFT}\left\{\sum_{v=1}^{V} b_{v} X_{v}\right\}=\sum_{v=1}^{V} b_{v} . \operatorname{IFFT}\left\{X_{v}\right\}=\sum_{v=1}^{V} b_{v} x_{v}
$$

where the phase factor $b=\left\{b_{v}=e^{j 2 \pi v / W} \mid v=0,1, \ldots, W-1\right\}$ , and $W$ is the number of the allowed phase factors. The first element of the phase factors $b_{1}$ usually set to 1 , without loss of PAPR performance. Moreover, the different number of phase factors $W$ is usually constant, so that $b_{v} \in\{ \pm 1\}$ or $\{ \pm 1, \pm \mathrm{j}\}$ to avoid complex multiplication operations [33].

In general, there are $W^{V-I}$ sets of the phase factors should also be searched to find the optimum phase factor and, the transmitter should send bits as side information (SI) to the receiver in order to recover the original data [18].

\section{PTS SEGMENTATION SCHEMES}

In PTS method, there are three common types of the segmentation schemes, including interleaving partition, 
adjacent partition and, pseudo-random partition as shown in Fig. 2. In interleaving segmentation scheme (IL-PTS), $N / V$ subcarriers are allocated within a certain distance interval of $V$ for each subblock. The adjacent segmentation scheme (AdPTS) allocates $N / V$ successive subcarriers within subblocks sequentially. The pseudo-random segmentation scheme (PRPTS) assigns the subcarriers randomly in the subblocks. The segmentation schemes must be under the following conditions: the subblocks should be equally in size, and the subcarrier must appear only one time within the subblock. Moreover, the subblocks must be non-overlapping with each other [34].
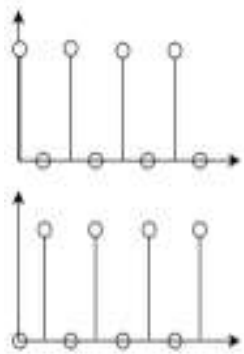

Interleaving
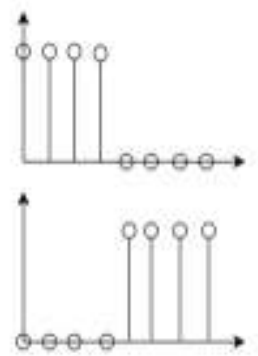

Adjacent
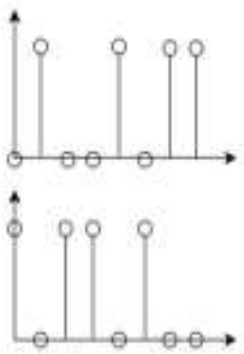

Pseuso-random
Fig. 2. Ordinary PTS segmentation schemes

The three types of partitioning influence on the PAPR reduction performance, which depend on the autocorrelation between the subcarriers. PR-PTS scheme can achieve the best PAPR performance among partitioning schemes, but the cost is an increased complexity. Ad-PTS scheme is lower PAPR reduction gain than PR-PTS scheme. In contrast, IL-PTS scheme considers the worst PAPR reduction performance, but its computational complexity is lower than other methods [30].

\section{PROPOSED METHODS}

\section{A. Symmetrical Interleaving Scheme-Cosine Wave Shape}

As mention, IL-PTS is one of the segmentation schemes, and its PAPR performance considered as the worse than the other schemes. However, the computational complexity of ILPTS is lower than that of Ad-PTS and PR-PTS scheme. The symmetrical interleaving scheme cosine wave shape PTS method (S-IL-C-PTS) can improve the PAPR reduction performance better than IL-PTS and Ad-PTS without increasing the computational complexity.

Fig. 3 illustrates the proposed method, which begins with segmenting the data sequence into $V$ subblocks similar to that of the IL-PTS scheme. After that, the IL-PTS matrix divided into $\mathrm{S}_{\mathrm{G}}$ groups, where $\mathrm{G}=\{1,2,3, \ldots, N / V\}$, and each group has $V$ rows. Then, only the even $\mathrm{S}_{\mathrm{G}}$ groups from the IL-PTS matrix are chosen for processing. Afterward, in each even $\mathrm{S}_{\mathrm{G}}$ groups, the first row is changed with last one and the second row with the one before the last, and so on. The IL-PTS matrix is changed into a new matrix, in which the subcarriers are allocated symmetrically as cosine wave shape. Finally, the procedure of PTS technique is applied to the proposed partitioning scheme, and the OFDM signal with the lowest PAPR value is chosen for transmission. The S-IL-C-PTS scheme can decrease the autocorrelation among the subcarriers within the subblocks. Hence, the PAPR reduction performance will be enhanced accordingly.
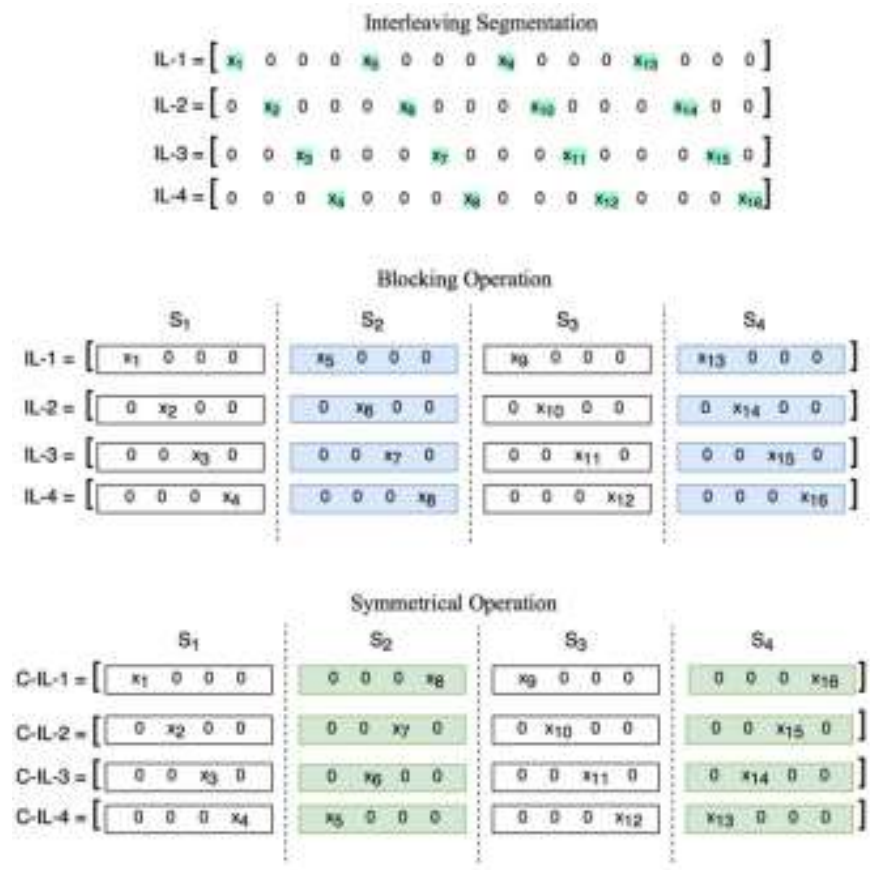

$$
\begin{aligned}
& \text { Symunetrical Interieaving Segmentation } \\
& C-1 t-1=\left[\begin{array}{llllllllllllllll}
x_{4} & 0 & 0 & 0 & 0 & 0 & 0 & x_{0} & x_{3} & 0 & 0 & 0 & 0 & 0 & 0 & x_{16}
\end{array}\right] \\
& C-1 L-2=\left[\begin{array}{llllllllllllllll}
0 & s_{2} & 0 & 0 & 0 & 0 & x_{7} & 0 & 0 & x_{i i} & 0 & 0 & 0 & 0 & x_{18} & 0
\end{array}\right]
\end{aligned}
$$

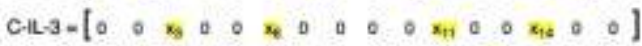

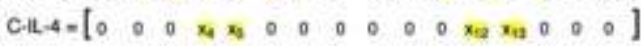

Fig. 3. S-IL-C-PTS scheme when $\mathrm{V}=4$ and $\mathrm{N}=16$

\section{B. Symmetrical Interleaving Scheme-Sine Wave Shape}

The symmetrical interleaving sine wave shape PTS method (S-IL-S-PTS) is the same as that of S-IL-C-PTS, but the difference is the S-IL-S-PTS scheme deals with the odd $\mathrm{S}_{\mathrm{G}}$ groups only, while the S-IL-C-PTS method is processed with the even $\mathrm{S}_{\mathrm{G}}$ groups from the Interleaving matrix.

Fig. 4 shows the S-IL-S-PTS segmentation operation, in which the input data $X$ is partitioned into disjoint subsets to generate the interleaving matrix. After that, the interleaving matrix is split into $\mathrm{S}_{\mathrm{G}}$ groups, and each $\mathrm{S}_{\mathrm{G}}$ group contains $V$ rows, where $\mathrm{G}=\{1,2,3, \ldots, N / V\}$. Afterward, only the odd $\mathrm{S}_{\mathrm{G}}$ groups are chosen for processing, and then the first row is changed with the last one and the second row with the one before the last, etc. lastly, the S-IL-S-PTS segmentation matrix is obtained, and the subcarriers allocated symmetrically, similar to the sine wave shape.

The S-IL-S-PTS segmentation is applied to PTS algorithm, and the OFDM signal with the lowest PAPR value is selected for transmission. The S-IL-S-PTS method works to reduce the autocorrelation between the subcarriers of the subblocks. Therefore, the PAPR reduction performance is improved accordingly. Moreover, the computational complexity of the SIL-S-PTS is the same as that of the IL-PTS method. 
Interleaving Segmentation

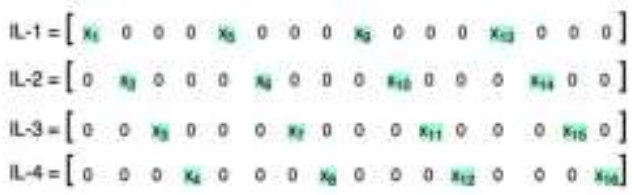

Blocking Operation
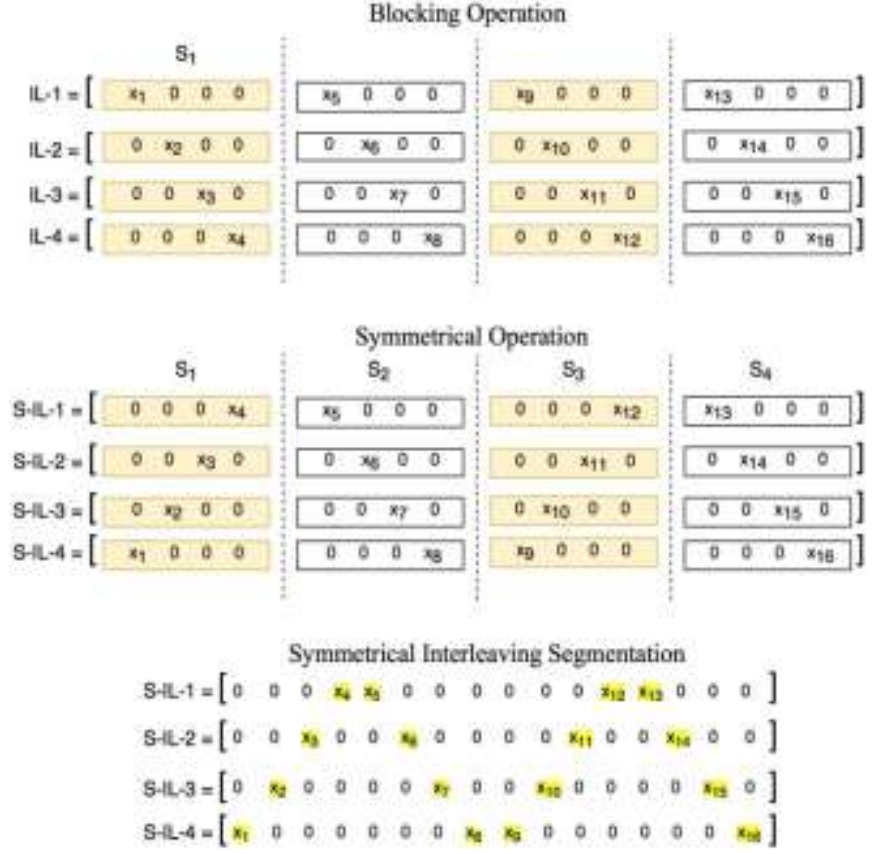

Fig. 4. S-IL-S-PTS scheme when $\mathrm{V}=4$ and $\mathrm{N}=16$

\section{Hybrid adjacent and interleaving segmentation PTS}

Ad-PTS is one of the segmentation methods for PTS algorithm, in which the successive subcarriers are assigned within subblocks sequentially. This approach can be achieved PAPR reduction performance better than that of IL-PTS, but the computational complexity is higher than that of IL-PTS.

Hybrid adjacent and interleaving segmentation PTS method (H-Ad-IL-PTS) is a new hybrid scheme that combines the features of both adjacent partitioning and interleaving partitioning schemes. H-Ad-IL-PTS can improve the PAPR reduction performance better than of both Ad-PTS and IL-PTS schemes. Moreover, its computational complexity is the same as that of IL-PTS scheme.

Fig. 5 illustrates the H-Ad-IL-PTS where the input data sequence is partitioned into $V$ subblocks by using Ad-PTS method. Then, each row of the adjacent matrix is sub-divided into $\mathrm{B}_{\mathrm{G}}$ blocks, and each block contains $V / 2$ subcarriers, where $\mathrm{G}=\{1,2, \ldots, N /(V / 2)\}$. Afterward, the interleaving method is applied to the modified adjacent matrix; with the consideration that; the original subcarriers of each $\mathrm{S}_{\mathrm{G}}$ block are kept in the same positions. Finally, the obtained H-Ad-IL-PTS scheme is fed to PTS algorithm to generate the OFDM signal. The proposed scheme exploits the capability of the Ad-PTS for reducing the PAPR performance and the tendency of the ILPTS for decreasing the computational complexity. Therefore, H-Ad-IL-PTS method outperforms to the Ad-PTS and IL-PTS methods.

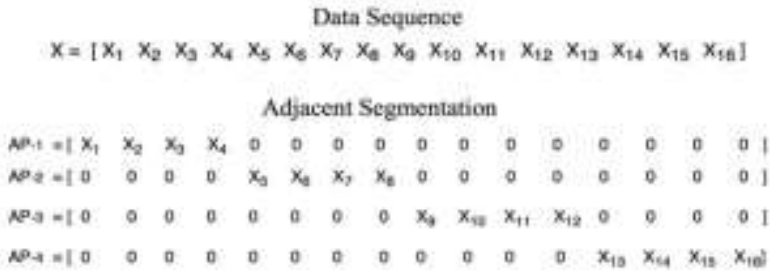

Blocking Operution

\begin{tabular}{|c|c|c|c|c|c|c|c|c|c|c|c|c|c|c|c|}
\hline 3 & $x_{1} \quad x$ & & $x_{4}$ & 0 & & a & 0 & 0 & 0 & 0 & 0 & 0 & 0 & 0 & 0 \\
\hline 2 & 0 & 0 & 0 & $x_{2}$ & $x_{t}$ & $x_{r}$ & $x_{0}$ & 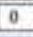 & 0 & 9 & $\overline{0}$ & 0 & 0 & 8 & 0 \\
\hline 1 & 0 & 0 & 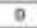 & 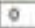 & 0 & 0 & 0 & $x_{3}$ & $x_{10}$ & $x_{11}$ & $x_{12}$ & 0 & 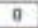 & 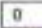 & 0 \\
\hline 34 & 10 & 0 & 0 & 0 & f & 0 & & 0 & & 0 & 0 & $x_{03}$ & $x_{i 4}$ & $x_{15}$ & $x_{+6}$ \\
\hline
\end{tabular}

\begin{tabular}{|c|c|c|c|c|c|c|c|c|c|c|c|c|c|c|c|c|}
\hline \multicolumn{17}{|c|}{ Interleaving Segmentation } \\
\hline are $=1$ & $x_{1}$ & $x_{2}$ & 0 & 0 & 0 & 0 & 0 & 0 & $x_{9}$ & $x_{10}$ & 0 & 0 & 品 & o & 0 & $\stackrel{\circ}{\circ}$ \\
\hline$A P-E=2=1$ & 0 & 0 & $x_{2}$ & $x_{6}$ & 0 & 0 & 0 & 0 & 0 & 0 & $x_{11}$ & $x_{12}$ & $\theta$ & 0 & 0 & 0 \\
\hline NPES $=1$ & 0 & 0 & 0 & 0 & $x_{0}$ & $x_{4}$ & 0 & 0 & 0 & 0 & 0 & a & $x_{13}$ & $x_{m a}$ & 0 & 0 \\
\hline AP-R $+=1$ & 0 & 0 & $a$ & $\circ$ & 0 & 0 & $x_{2}$ & $x_{n}$ & 0 & 0 & 0 & 0 & 0 & 0 & $x_{48}$ & $x_{18}$ \\
\hline
\end{tabular}

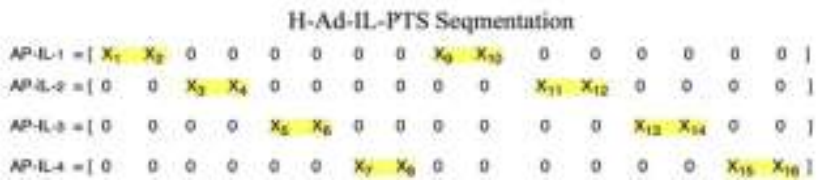

Fig. 5. H-Ad-IL-PTS scheme when $V=4$ and $N=16$

\section{Symmetrical H-Ad-IL-PTS Cosine Wave Shape}

The basic algorithm of the symmetrical H-Ad-IL-PTS cosine wave shape (SC-H-Ad-IL-PTS) is a combination of HAd-IL-PTS scheme and S-IL-C-PTS scheme. This method works to break down the autocorrelation between the subcarriers within the subblocks. Thus the PAPR reduction rendering will be improved accordingly. In addition, the computational complexity of the proposed scheme is the same as that of the IL-PTS method.

Fig. 6 clarifies the SC-H-Ad-IL-PTS scheme, in which the H-Ad-IL-PTS matrix is applied to the S-IL-C-PTS algorithm to generate the new scheme. The procedure is division the H-AdIL-PTS matrix into $\mathrm{B}_{\mathrm{G}}$ groups, where $\mathrm{G}=\{1,2, \ldots, N / 2 V\}$. After that, only even groups are selected for processing. In every even group, the first row is changed with the last one and the second row with the one before the last, and so on. Finally, the proposed scheme is implemented on the PTS procedure, and the OFDM signal that has the lowest PAPR value is elected for transmission. 


\section{H-Ad-IL-PTS Segmentation}

\begin{tabular}{|c|c|c|c|c|c|c|c|c|c|c|c|c|c|c|c|}
\hline$A=\mid L-1=1 X_{1}$ & $x_{2}$ & 0 & 0 & 0 & 0 & 0 & 0 & $x_{3}$ & $x_{v t}$ & 0 & 0 & 0 & 0 & 0 & \\
\hline APLL-2 $=10$ & 0 & $x_{3}$ & $x_{4}$ & 0 & 0 & 0 & 0 & 0 & 0 & $x_{11}$ & $x_{12}$ & 0 & 0 & 0 & 0 \\
\hline AP- 1 L $-a=[0$ & 0 & 0 & 0 & $x_{3}$ & $x_{6}$ & 0 & 0 & 0 & 0 & 0 & 0 & $x_{13}$ & $x_{14}$ & 0 & a \\
\hline$A P-1 L-1=10$ & 0 & 0 & 0 & 0 & 0 & $x_{7}$ & $x_{1}$ & 0 & 0 & 0 & 0 & 0 & 0 & $x_{19}$ & $x_{18}$ \\
\hline
\end{tabular}

\begin{tabular}{|c|c|c|c|c|c|c|c|c|c|c|c|c|c|c|c|}
\hline \multirow[b]{3}{*}{$A P L-1=1$} & \multicolumn{15}{|c|}{ Symmetrical Cosine Shape Operation } \\
\hline & \multicolumn{7}{|c|}{$\theta_{9}$} & \multicolumn{6}{|c|}{$B_{2}$} & \multirow[b]{2}{*}{0} & \multirow[b]{2}{*}{01} \\
\hline & $x_{2}$ & 0 & 0 & 0 & 0 & 0 & 0 & $x_{9}$ & $x_{i b}$ & 0 & 0 & 0 & 0 & & \\
\hline AP:H-E $=10$ & 0 & $x_{2}$ & $\bar{x}_{4}$ & 0 & 0 & 0 & 0 & 0 & 0 & $x_{11}$ & $x_{12}$ & 0 & 0 & 0 & 01 \\
\hline$A P-A=10$ & 0 & 0 & 2 & $x_{s}$ & $x_{s}$ & 6 & 0 & 0 & 0 & - & a & $x_{13}$ & $x_{14}$ & 0 & 0.1 \\
\hline APAt $=0$ & 0 & 0 & 0 & 9 & $\theta$ & $x_{t}$ & $x_{2}$ & 0 & 0 & 0 & 0 & 0 & 0 & & $\left.x_{10}\right]$ \\
\hline
\end{tabular}
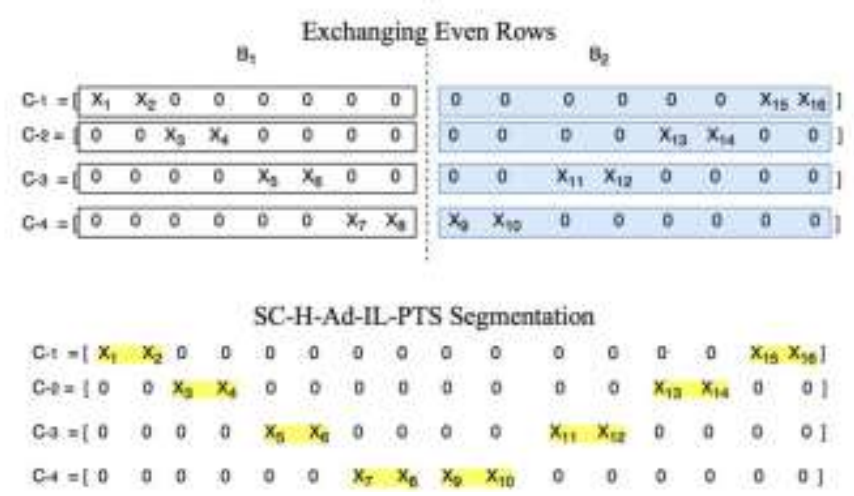

Fig. 6. SC-H-Ad-IL-PTS scheme when $\mathrm{V}=4$ and $\mathrm{N}=16$

\section{E. Symmetrical H-Ad-IL-PTS Sine Wave Shape}

\section{H-Ad-IL-PTS Segmentation}

\begin{tabular}{|c|c|c|c|c|c|c|c|c|c|c|c|c|c|c|c|}
\hline$A P E-1=\mid X_{1}$ & $x_{2}$ & 0 & 0 & 0 & 0 & 0 & 0 & $x_{6}$ & $x_{10}$ & 0 & 0 & 0 & 0 & 0 & \\
\hline$A P U L=2=10$ & 0 & $x_{3}$ & $x_{4}$ & 0 & 0 & 0 & 0 & 0 & 0. & $x_{15}$ & $x_{12}$ & o & 0 & o & 0 \\
\hline APLS $=10$ & 0 & 0 & 0 & $x_{6}$ & $x_{4}$ & 0 & 0 & 0 & 0 & 0 & 0 & $x_{13}$ & $x_{14}$ & 0 & 0 \\
\hline$A P-24=10$ & 0 & 0 & 0 & 0 & 0 & $x_{7}$ & $x_{6}$ & 0 & 0 & 0 & 0 & 0 & 0 & $x_{48}$ & \\
\hline
\end{tabular}
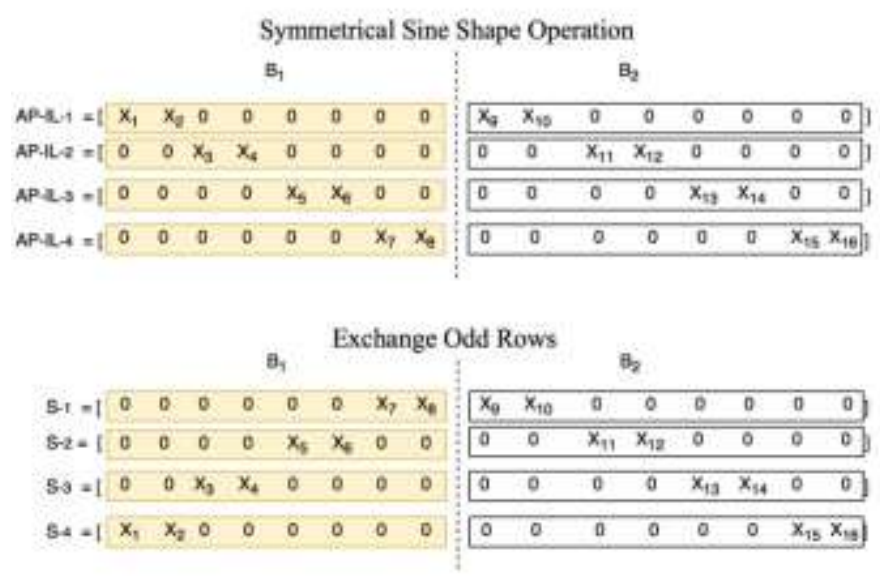

SS-H-Ad-IL-PTS Segmentation

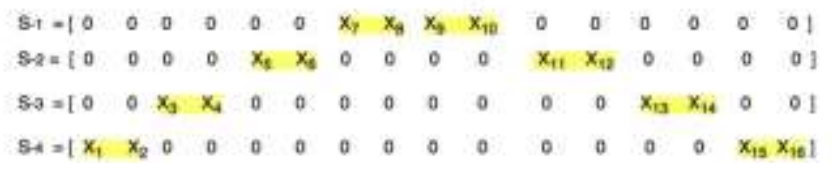

Fig. 7. SS-H-Ad-IL-PTS scheme when $\mathrm{V}=4$ and $\mathrm{N}=16$
This method merges the H-Ad-IL-PTS scheme with S-ILS-PTS scheme in the frequency domain to produce a new scheme named symmetrical H-Ad-IL-PTS sine wave shape (SS-H-Ad-IL-PTS). The proposed method can diminish the PAPR value better than Ad-PTS and IL-PTS because the autocorrelation between the subcarriers is decreased. Moreover, SS-H-Ad-IL-PTS method can achieve computational complexity less than that of the Ad-PTS method and the same computational complexity of the IL-PTS method.

The proposed method is shown in Fig. 7, where the H-AdIL-PTS is established, and then the matrix is divided into $B_{G}$ groups, where $\mathrm{G}=\{1,2, \ldots, N / 2 \mathrm{~V}\}$. Afterward, only the odd $\mathrm{B}_{\mathrm{G}}$ groups are selected from the H-IL-Ad-PTS matrix for processing, in which the first row of the odd $\mathrm{B}_{\mathrm{G}}$ groups is changed with the last row and the second row with the one preceding the last, etc. Lastly, the PTS pattern performs on the new scheme to create a set of candidate signals. The candidate signal which can realize the lower PAPR value is selected for transmission.

\section{RESULTS AND DISCUSSION}

In this section, a computer simulation has been implemented to evaluate and analysis the proposed methods and ordinary PTS method. The simulation parameters that used in this simulation are: the number of subcarriers $N=128$ and 256 , the number of subblocks $V=4$, the number of allowed phase rotation factors $W=4$ and the data samples are mapped by 16-QAM. In addition, 1000 OFDM symbols are generated randomly to evaluate the PAPR performance, and the oversampling factor is set to 8 .

Firstly, the three common types of the segmentation schemes are simulated when $N=128$ and 256, as shown in Fig. 8 and Fig. 9, respectively. As can be seen from Fig. 8, the PAPR reduction rendering of the PR-PTS scheme is superior to Ad-PTS, IL-PTS and the original OFDM signal by $0.84 \mathrm{~dB}$, $1.27 \mathrm{~dB}$, and $4 \mathrm{~dB}$, respectively. Moreover, Fig. 9 shows the PAPR performance of the PR-PTS, Ad-PTS, IL-PTS, and the original signal when $N=256$. The CCDF of the PR-PTS achieved better PAPR reduction performance at $7.77 \mathrm{~dB}$ and Ad-PTS was the next best at $8.51 \mathrm{~dB}$. However, IL-PTS scheme realized the worst among the three segmentation schemes at $9.17 \mathrm{~dB}$.

In simulation result as shown in Fig. 10, the S-IL-C-PTS method is compared with Ad-PTS and IL-PTS methods when the $\mathrm{CCDF}=10^{-3}$ and $N=128$. The PAPR value of the original signal was $11 \mathrm{~dB}$, IL-PTS was $8.4 \mathrm{~dB}$, and Ad-PTS was $8.15 \mathrm{~dB}$. However, the S-IL-C-PTS algorithm can achieve better PAPR reduction performance at $7.45 \mathrm{~dB}$. In addition, Fig. 11 simulates the same parameters that conducted in Fig. 10 except the subcarriers number increased to 256. S-IL-C-PTS was also achieved greater PAPR performance at $8.1 \mathrm{~dB}$ compared with Ad-PTS and IL-PTS schemes. Therefore, S-IL-C-PTS scheme considers better performance than Ad-PTS and IL-PTS schemes for any number of the subcarriers.

Simulation comparison of PAPR reduction rendering is implemented by using S-IL-S-PTS scheme and Ad-PTS, ILPTS as well as the original signal, with the consideration that; the number of subcarriers $N$ is 128 and 256. It shown in Fig. 
12, the PAPR performance of the S-IL-S-PTS scheme is $7.44 \mathrm{~dB}$. However, the PAPR value of the Ad-PTS, IL-PTS, and the original signal is $8.15 \mathrm{~dB}, 8.40 \mathrm{~dB}$, and $11 \mathrm{~dB}$, respectively. Moreover, Fig. 13 shows the PAPR performance of the S-IL-S-PTS when the number of subcarriers increased to 256. It is clearly, the proposed method can minimize the PAPR value by $3.85 \mathrm{~dB}$ from the original OFDM signal. Therefore, the S-IL-S-PTS method is superior to Ad-PTS and IL-PTS schemes for various numbers of subcarriers.

The observation result as shown in Fig. 14, the new hybrid method is compared with Ad-PTS and IL-PTS when $N=128$. The H-Ad-IL-PTS algorithm achieved PAPR performance better than Ad-PTS and IL-PTS schemes by $0.71 \mathrm{~dB}$ and 0.96 $\mathrm{dB}$, respectively. In the same manner, the H-Ad-IL-PTS algorithm compared with adjacent and interleaving segmentation schemes when $N=256$, as shown in Fig. 15. The hybrid PTS method overcomes to the Ad-PTS and IL-PTS by $0.6 \mathrm{~dB}$ and $1.1 \mathrm{~dB}$, respectively. Therefore, the H-Ad-IL-PTS scheme can be realized greater PAPR reduction performance than other ordinary schemes with the same number of subcarriers.

As can be seen from Fig. 16 and Fig. 17, The SC-H-Ad-ILPTS method can reduce the PAPR value by $3.42 \mathrm{~dB}$ and $4 \mathrm{~dB}$ compared with the original OFDM signal, when the number of subcarriers is fixed at 128 and 256, respectively. Therefore, the enhanced method can improve the PAPR performance more valuable than Ad-PTS and IL-PTS schemes.

Similarly, Fig. 18 and Fig. 19 presents the simulation result for SS-H-Ad-IL-PTS algorithm compared with Ad-PTS and IL-PTS schemes when the subcarriers number is 128 and 256. The enhanced method can get better PAPR performance than Ad-PTS by $0.77 \mathrm{~dB}$ and IL-PTS by $1.12 \mathrm{~dB}$ when $N=128$. However, the PAPR performance of the SS-H-Ad-IL-PTS method is greater than the Ad-PTS and IL-PTS schemes by $0.7 \mathrm{~dB}$ and $1.2 \mathrm{~dB}$ when the number of subcarriers is 256 .

TABLE I. NUMERICAL SiMULATION RESULTS OF THE VARIOUS SEGMENTATION SCHEMES

\begin{tabular}{|l|l|l|}
\hline V=4, $\mathbf{W}=\mathbf{4}$ & PAPR[dB] \\
\cline { 2 - 3 } PTS Methods & $\mathbf{N = 1 2 8}$ & $\mathbf{N = 2 5 6}$ \\
\hline Original signal & 11 & 12.05 \\
\hline Ad-PTS & 8.15 & 8.55 \\
\hline IL-PTS & 8.4 & 9.05 \\
\hline S-IL-C-PTS & 7.45 & 8.1 \\
\hline S-IL-S-PTS & 7.44 & 8.1 \\
\hline H-Ad-IL-PTS & 7.58 & 8.1 \\
\hline SC- H-Ad-IL-PTS & 7.28 & 7.85 \\
\hline SS- H-Ad-IL-PTS & 7.26 & 7.76 \\
\hline
\end{tabular}

Table I summarizes all the numerical simulation results. It is clearly, the proposed methods can be achieved greater PAPR reduction rendering than the ordinary methods. In addition, Table II and Table III demonstrate the PAPR reduction ratio according to the original OFDM signal for different PTS schemes. It can be concluded that the proposed schemes can achieve better PAPR reduction percentages than the conventional schemes IL-PTS and IL-PTS in both scenarios. On the other hand, the proposed methods can improve the PAPR performance without any increasing in complexity.

TABLE II. PAPR REDUCTION RATIO OF THE VARIOUS SEgMENTATION SCHEMES WHEN $\mathrm{N}=128$

\begin{tabular}{|c|c|c|c|}
\hline \multicolumn{4}{|c|}{$V=4, W=4, N=128$} \\
\hline PTS Methods & $\begin{array}{l}\text { PAPR of } \\
\text { Original } \\
\text { Signal } \\
{[d B]}\end{array}$ & $\begin{array}{l}\text { PAPR } \\
\text { of PTS } \\
\text { Method } \\
\text { [dB] }\end{array}$ & $\begin{array}{l}\text { PAPR } \\
\text { Reduction } \\
\text { Ratio }\end{array}$ \\
\hline Ad-PTS & \multirow{7}{*}{11} & 8.15 & $25.9 \%$ \\
\hline IL-PTS & & 8.4 & $23.63 \%$ \\
\hline S-IL-C-PTS & & 7.45 & $32.27 \%$ \\
\hline S-IL-S-PTS & & 7.44 & $32.36 \%$ \\
\hline H-Ad-IL-PTS & & 7.58 & $31.09 \%$ \\
\hline SC-H-Ad-IL-PTS & & 7.28 & $33.81 \%$ \\
\hline SS-H-Ad-IL-PTS & & 7.26 & $34 \%$ \\
\hline
\end{tabular}

TABLE III. PAPR REDUCTION RATIO OF THE VARIOUS SEGMENTATION SCHEMES WHEN $\mathrm{N}=256$

\begin{tabular}{|c|c|c|c|}
\hline \multicolumn{4}{|c|}{$\mathrm{V}=4, \mathrm{~W}=4, \mathrm{~N}=256$} \\
\hline PTS Method & $\begin{array}{l}\text { PAPR of } \\
\text { Original } \\
\text { Signal } \\
{[d B]}\end{array}$ & $\begin{array}{l}\text { PAPR } \\
\text { of PTS } \\
\text { Method } \\
{[\mathrm{dB}]}\end{array}$ & $\begin{array}{l}\text { PAPR } \\
\text { Reduction } \\
\text { Ratio }\end{array}$ \\
\hline Ad-PTS & \multirow{7}{*}{12} & 8.55 & $28.75 \%$ \\
\hline IL-PTS & & 9.05 & $24.58 \%$ \\
\hline S-IL-C-PTS & & 8.1 & $32.5 \%$ \\
\hline S-IL-S-PTS & & 8.1 & $32.5 \%$ \\
\hline H-Ad-IL-PTS & & 8.1 & $32.5 \%$ \\
\hline SC-H-Ad-IL-PTS & & 7.85 & $34.58 \%$ \\
\hline SS-H-Ad-IL-PTS & & 7.76 & $35.33 \%$ \\
\hline
\end{tabular}




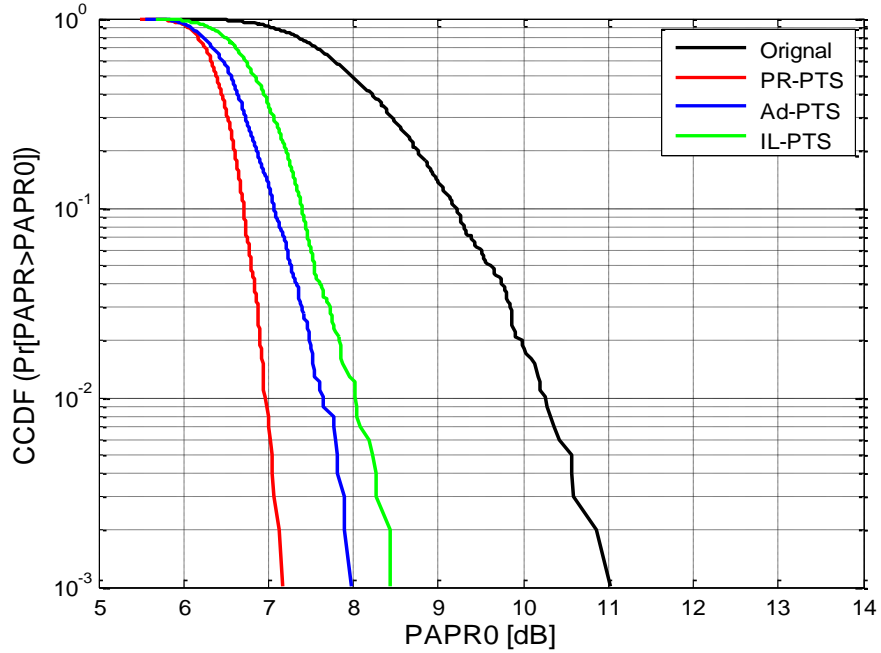

Fig. 8. PAPR comparison of the three ordinary PTS segmentation schemes for $\mathrm{N}=128$

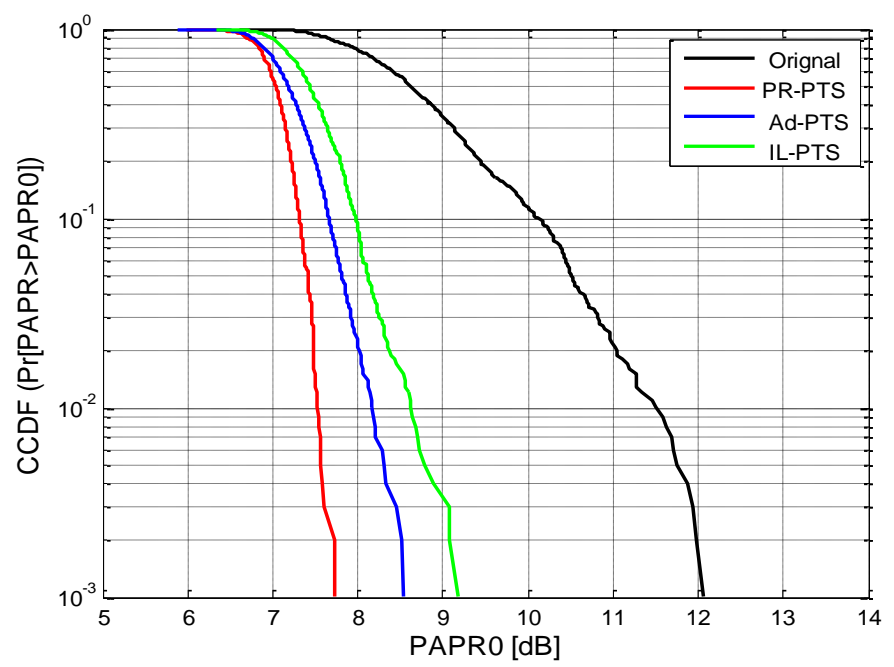

Fig. 9. PAPR comparison of the three ordinary PTS segmentation schemes for $\mathrm{N}=256$

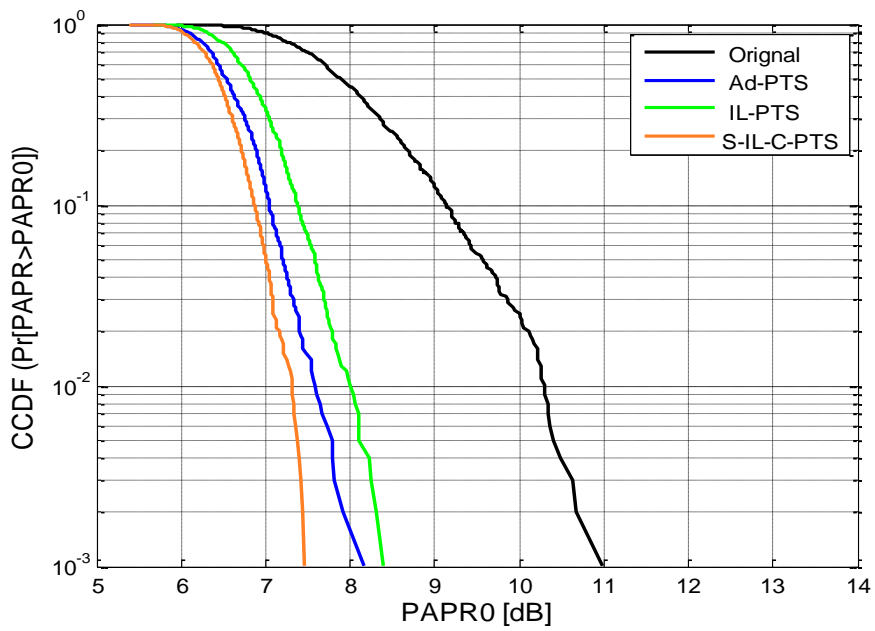

Fig. 10. PAPR comparison of the S-IL-C-PTS scheme and the ordinary PTS schemes for $\mathrm{N}=128$

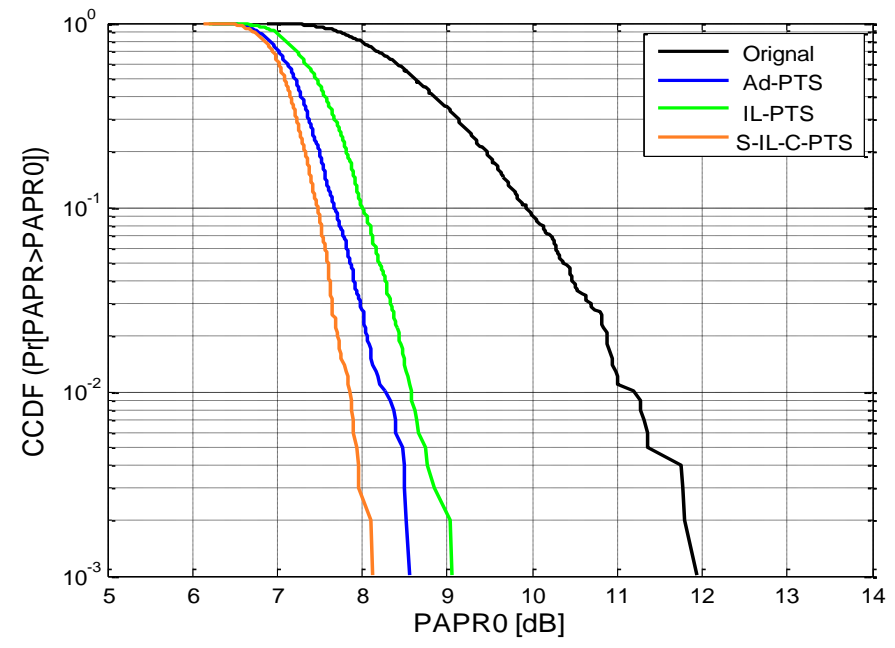

Fig. 11. PAPR comparison of the S-IL-C-PTS scheme and the ordinary PTS schemes for $\mathrm{N}=256$

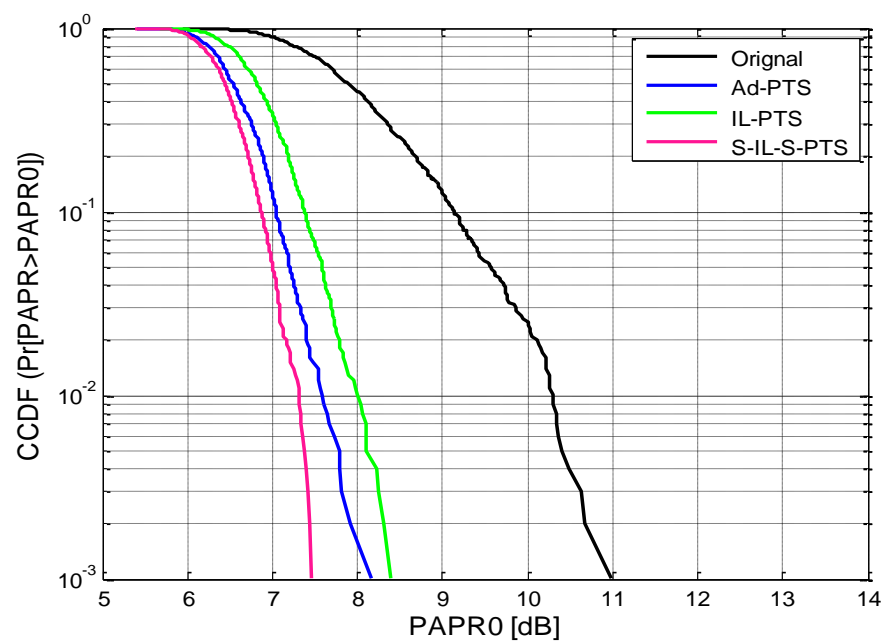

Fig. 12. PAPR comparison of the S-IL-S-PTS scheme and the ordinary PTS schemes for $\mathrm{N}=128$

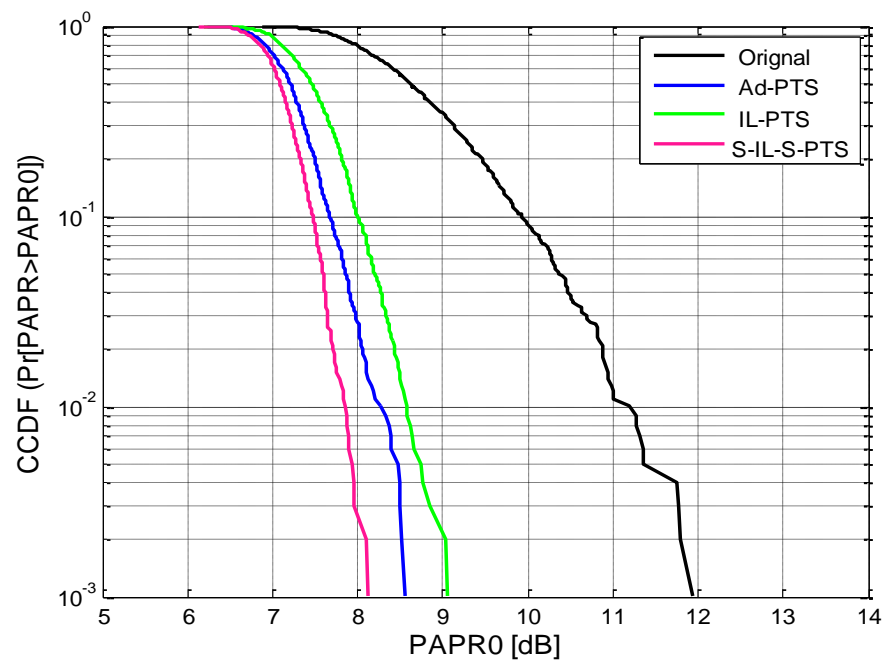

Fig. 13. PAPR comparison of the S-IL-S-PTS scheme and the ordinary PTS schemes for $\mathrm{N}=256$ 


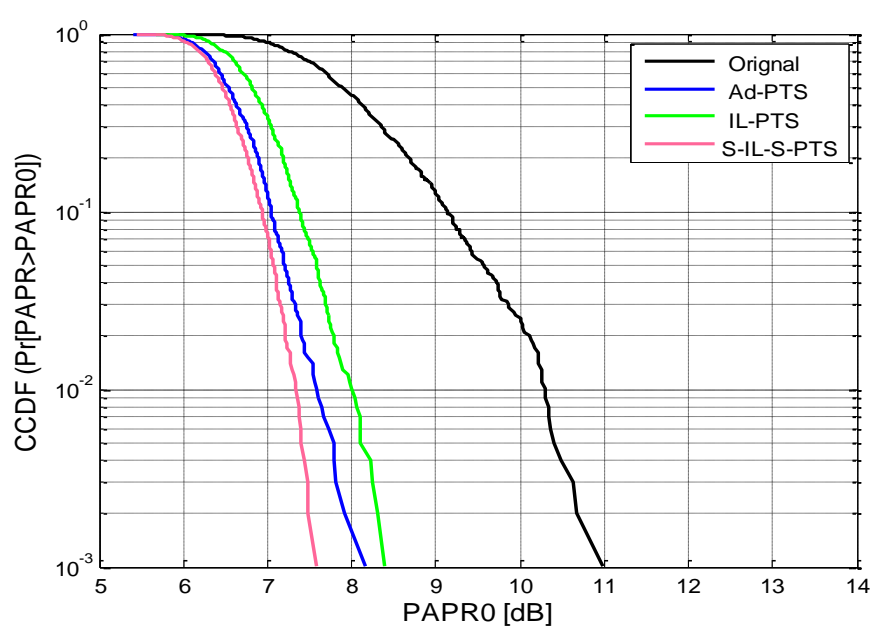

Fig. 14. PAPR comparison of the H-Ad-IL-PTS scheme and the ordinary PTS schemes for $\mathrm{N}=128$

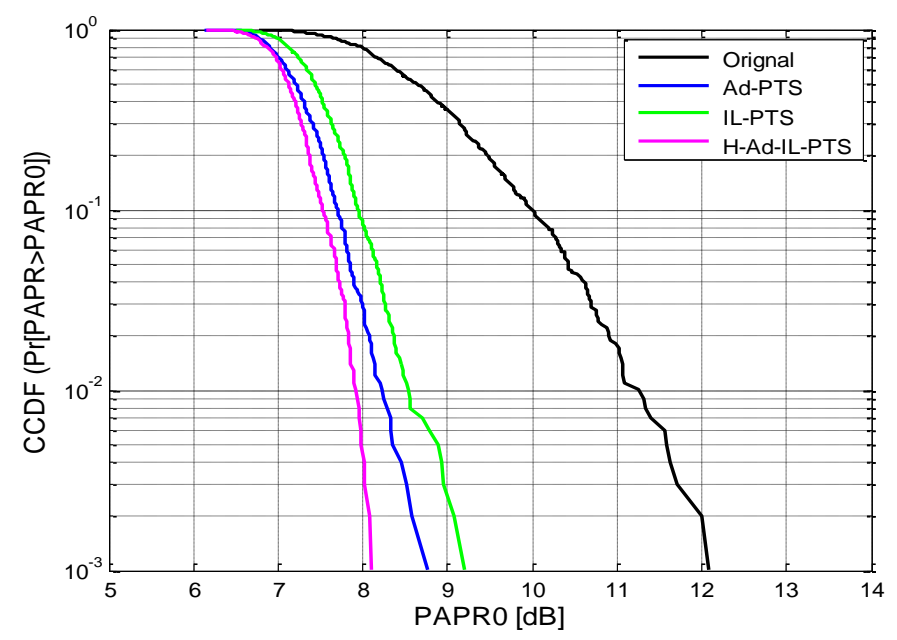

Fig. 15. PAPR comparison of the H-Ad-IL-PTS scheme and the ordinary PTS schemes for $\mathrm{N}=256$

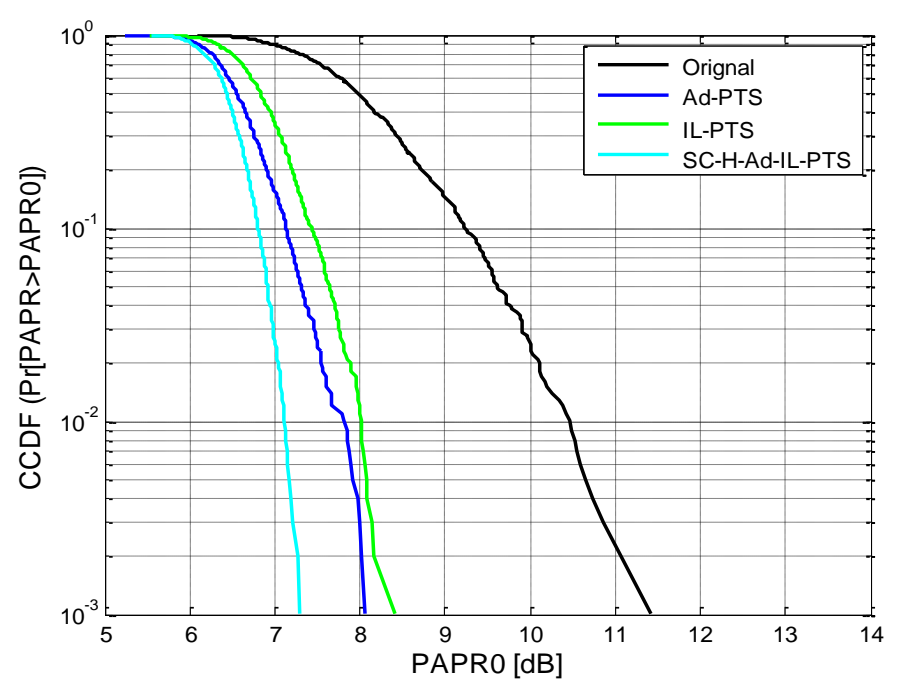

Fig. 16. PAPR comparison of the SC-H-Ad-IL-PTS scheme and thee ordinary PTS schemes for $\mathrm{N}=128$

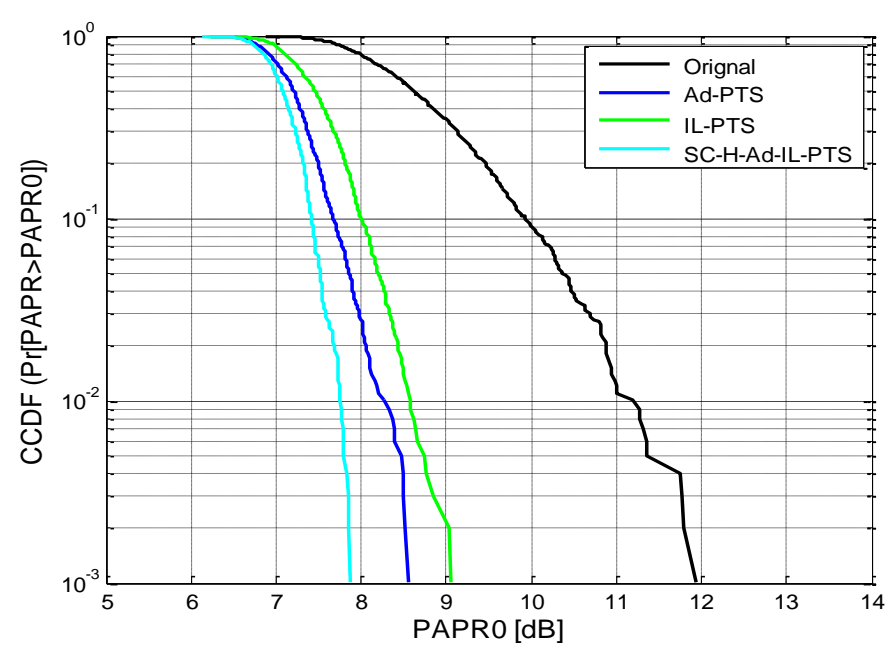

Fig. 17. PAPR comparison of the SC-H-Ad-IL-PTS scheme and the ordinary PTS schemes for $\mathrm{N}=256$

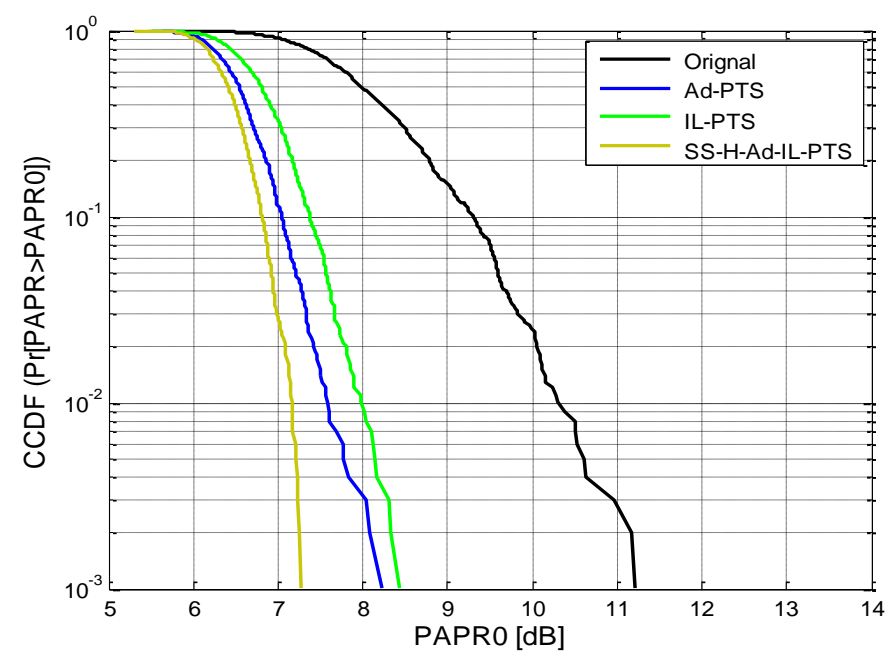

Fig. 18. PAPR comparison of the SS-H-Ad-IL-PTS scheme and the ordinary PTS schemes for $\mathrm{N}=128$

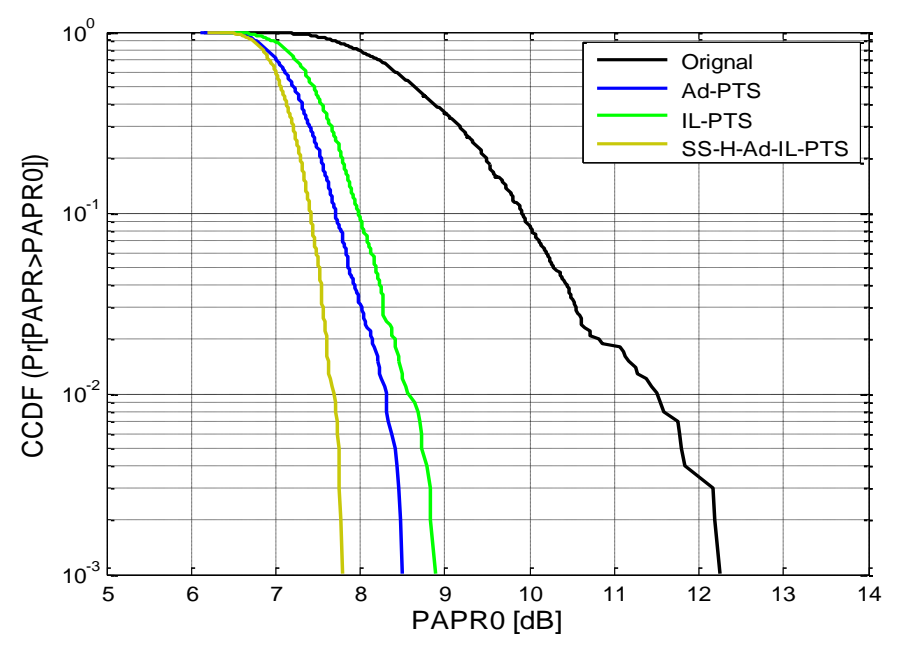

Fig. 19. PAPR comparison of the SS-H-Ad-IL-PTS scheme and the ordinary PTS schemes for $\mathrm{N}=256$ 


\section{CONCLUSION}

In this paper, the new PTS segmentation methods to reduce the PAPR in OFDM systems are proposed. The subblocks partition matrix of the PTS scheme is employed to generate the new types of the of segmentation schemes including S-IL-CPTS, S-IL-S-PTS, H-Ad-IL-PTS, SC-H-Ad-IL-PTS, and SSH-Ad-IL-PTS. The simulation results show the proposed methods can improve up the PAPR reduction execution compared with two well-known segmentation schemes adjacent and interleaving partition schemes. It has been seen that the proposed approaches can enhance the PAPR performance with any number of subcarriers, and the computational complexity of the new methods is kept low similar as that of the interleaving segmentation method. Therefore, the proposed schemes can be made the PTS technique more suitable for high-speed data rate wireless system, and the proposed methods could be applying in MIMO-OFDM for future work.

\section{REFERENCES}

[1] K. Pachori and A. Mishra, "An efficient combinational approach for PAPR reduction in MIMO-OFDM system," Wireless Networks, vol. 22, pp. 417-425, 2016.

[2] P. Praveenkumar, R. Amirtharajan, K. Thenmozhi, and J. B. B. Rayappan, "OFDM with low PAPR: A novel role of partial transmit sequence," Res. J. Inform. Technol, vol. 5, pp. 35-44, 2013.

[3] Z. Chen and S. G. Kang, "A three-dimensional OFDM system with PAPR reduction method for wireless sensor networks," International Journal of Distributed Sensor Networks, vol. 2014, pp.1-6, 2014.

[4] M. Vidya, M. Vijayalakshmi, and K. Ramalingareddy, "Performance enhancement of efficient partitioning technique for PAPR reduction in MIMO-OFDM system using PTS," 2015 Conference on Power, Control, Communication and Computational Technologies for Sustainable Growth (PCCCTSG), pp. 247-253, 2015.

[5] Y. A. Jawhar, R. A. Abdulhasan, and K. N. Ramli, "Influencing Parameters in Peak to Average Power Ratio Performance on Orthogonal Frequency-Division Multiplexing System," ARPN journal of engineering and applied sciences, vol. 11(7), pp. 4322-4332, 2016.

[6] B. H. Alhasson and M. A. Matin, "PAPR distribution analysis of OFDM signals with partial transmit sequence," 14th International Conference on in Computer and Information Technology (ICCIT), pp. 652-656, 2011.

[7] F.-S. FET-SSGI, "A Novel Approach to Reduce PAPR in OFDM Using combined DCT Precoding Technique and Clipping Method,"ARPN journal of engineering and applied sciences, vol. 10(5), pp. 2182-2186, 2015.

[8] L. Yang, K. K. Soo, S. Li, and Y. M. Siu, "PAPR reduction using low complexity PTS to construct of OFDM signals without side information," IEEE Transactions on Broadcasting, vol. 57, pp. 284-290, 2011.

[9] M. I. Abdullah, "Comparative study of PAPR reduction techniques in OFDM," ARPN journal of systemes and software, vol. 1(8), pp. 263269, 2011.

[10] Yasir Amer Jawhar, Raed A. Abdulhasan and Khairun Nidzam Ramli, "A New Hybrid Sub-Block Partition Scheme of PTS Technique for Reduction PAPR Performance in Ofdm System," ARPN journal of engineering and applied sciences, vol. 11(6), pp. 3904-3910, 2016.

[11] S. P. Mohammad and K. Gopal, "Hybrid Technique for BER and Paper Analysis of OFDM Systems," Indian Journal of Science and Technology, vol. 9(15), pp. 1-5, 2016.

[12] Z. T. Ibraheem, M. M. Rahman, S. Yaakob, M. S. Razalli, F. Salman, and K. K. Ahmed, "PTS Method with Combined Partitioning Schemes for Improved PAPR Reduction in OFDM System," Indonesian Journal of Electrical Engineering and Computer Science, vol. 12, pp. 7845-7853, 2014.
[13] X. Wu, J. Wang, Z. Mao, and J. Zhang, "Conjugate interleaved partitioning PTS scheme for PAPR reduction of OFDM signals," Circuits, Systems and Signal Processing, vol. 29, pp. 499-514, 2010.

[14] D. W. Lim, S. J. Heo, and J. S. No, "An overview of peak-to-average power ratio reduction schemes for OFDM signals," Communications and Networks Journal, vol. 11, pp. 229-239, 2009.

[15] M. A. Taher, J. Mandeep, M. Ismail, S. A. Samad, and M. T. Islam, "Reducing the power envelope fluctuation of OFDM systems using side information supported amplitude clipping approach," International Journal of Circuit Theory and Applications, vol. 42, pp. 425-435, 2014.

[16] L. Wang and C. Tellambura, "An overview of peak-to-average power ratio reduction techniques for OFDM systems," 2006 IEEE International Symposium on Signal Processing and Information Technology, pp. 840$845,2006$.

[17] T. Jiang and Y. Wu, "An overview: peak-to-average power ratio reduction techniques for OFDM signals," IEEE transactions on Broadcasting, vol. 54, pp. 257-268, 2008.

[18] S. H. Müller and J. B. Huber, "A comparison of peak power reduction schemes for OFDM," IEEE Global Telecommunications Conference, GLOBECOM'97, pp. 1-5, 1997.

[19] M. A. Taher, M. J. Singh, M. Ismail, S. A. Samad, M. T. Islam, and H. F. Mahdi, "Post-IFFT-Modified Selected Mapping to Reduce the PAPR of an OFDM System," Circuits, Systems, and Signal Processing, vol. 34, pp. 535-555, 2015.

[20] S. H. Müller and J. B. Huber, "OFDM with reduced peak-to-average power ratio by optimum combination of partial transmit sequences," Electronics letters, vol. 33, pp. 368-369, 1997.

[21] S. H. Han and J. H. Lee, "An overview of peak-to-average power ratio reduction techniques for multicarrier transmission," IEEE Wireless Communications, vol. 12, pp. 56-65, 2005.

[22] C. Hong, Q. Qin, and T. Chao, "An PTS optimization algorithm for PAPR reduction of OFDM system," 2013 International Conference on Mechatronic Sciences, Electric Engineering and Computer (MEC), pp. 3775-3778, 2013.

[23] L. Xia, X. Yue, L. Shaoqian, H. Kayama, and C. Yan, "Analysis of the performance of partial transmit sequences with different subblock partitions," 2006 International Conference on Communications, Circuits and Systems Proceedings, pp. 875-878, 2006.

[24] L. Xia, X. Yue, T. Youxi, and L. Shaoqian, "A novel method to design phase factor for PTS based on pseudo-random sub-block partition in OFDM system," 2007 IEEE 66th Vehicular Technology Conference, VTC-2007 Fall, pp. 1269-127, 2007.

[25] Y. Xiao, Q. Wen, X. Lei, and S. Li, "Improved PTS for PAPR reduction in OFDM systems," The Third Advanced International Conference on Telecommunications, AICT 2007, pp. 37-37, 2007.

[26] L. Miao and Z. Sun, "PTS algorithm for PAPR suppression of WOFDM system," Proceedings 2013 International Conference on Mechatronic Sciences, Electric Engineering and Computer (MEC), pp. 1144-1147, 2013.

[27] A. Singh and H. Singh, "Peak to average power ratio reduction in OFDM system using hybrid technique," Optik-International Journal for Light and Electron Optics, vol. 127, pp. 3368-3371, 2016.

[28] J. Zhou, E. Dutkiewicz, R. P. Liu, G. Fang, Y. Liu, and X. Huang, "A modified shuffled frog leaping algorithm for PAPR reduction in OFDM systems," IEEE Region 10 Conference TENCON 2014, pp. 1-6, 2014.

[29] M. Taher, M. Singh, M. Ismail, S. A. Samad, and M. T. Islam, "Sliding the SLM-technique to reduce the non-linear distortion in OFDM systems," Elektronika ir Elektrotechnika, vol. 19, pp. 103-111, 2013.

[30] L. Amhaimar, S. Ahyoud, A. Aselman, and E. Said, "Peak-to-Average Power ratio reduction based varied Phase for MIMO-OFDM Systems," International Journal of Advanced Computer Science and Applications, vol. 7, pp. 432-437, 2016.

[31] S. G. Kang, J. G. Kim, and E. K. Joo, "A novel subblock partition scheme for partial transmit sequence OFDM," IEEE Transactions on Broadcasting, vol. 45, pp. 333-338, 1999.

[32] L. Yang, R. S. Chen, Y. M. Siu, and K. K. Soo, "PAPR reduction of an OFDM signal by use of PTS with low computational complexity," IEEE Transactions on Broadcasting, vol. 52, pp. 83-86, 2006. 
[33] T. Jiang, W. Xiang, P. C. Richardson, J. Guo, and G. Zhu, "PAPR reduction of OFDM signals using partial transmit sequences with low computational complexity," IEEE Transactions on Broadcasting, vol. 53, pp. 719-724, 2007.
[34] L. Miao and Z. Sun, "PTS algorithm for PAPR suppression of WOFDM system," International Conference on Mechatronic Sciences, Electric Engineering and Computer (MEC), pp. 1144-1147, 2013. 